\title{
荷电理想流体球的内部度规
}

\author{
朱世昌 \\ (上海师范学院物理系)
}

荷电球对称的 Einstein-Maxwell 方程内部解是一类基本而重要的内部解,近年来不少作者 致力于此课题 ${ }^{[1-6]}$,大多数作者在均匀密度情况下 $e^{\lambda}=-g_{r r}$ 均取类似于下式的形式

$$
e^{\lambda}=\left(1-r^{2} / R_{0}^{2}-\frac{1}{5} h^{2} r^{4}\right)^{-1},
$$

式中 Schwarzschild 半径 $R_{0}^{2}=\frac{3}{8 \pi \rho_{0}},\left(\rho_{0}\right.$ 为质量密度 $) h=\frac{4 \pi}{3} \rho_{e}$ ( $\rho_{e}$ 为电荷密度). 本文证明 了式(1)不是共形平直的, 找到了共形平直的内部解, 并证明了此共形平直内部解满足共形唯 一性. 此外, 本文修正了由 Bekenstein ${ }^{[5]}$ 在 1971 年得到的荷电情况下推广的 Tolman-Oppenheimer-Volkoff 方程(以下简称 $\mathrm{T}-\mathrm{O}-\mathrm{V}$ 方程).

\section{一、内部解的一般形式和推广的 $\mathrm{T}-\mathrm{O}-\mathrm{V}$ 方程}

荷电理想流体在静态球对称情况下其 Einstein-Maxwell 方程取如下形式:

$$
\begin{gathered}
\frac{1}{\sqrt{-g}} \frac{\partial}{\partial x_{\nu}}\left(\sqrt{-g} f^{\mu \nu}\right)=4 \pi j^{\mu}, \\
G_{0}^{0}=e^{-\lambda}\left(\frac{1}{r^{2}}-\frac{\lambda^{\prime}}{r}\right)-\frac{1}{r^{2}}=-8 \pi \rho_{0}-E^{2}, \\
G_{1}^{1}=\frac{1}{r^{2}}-e^{-\lambda}\left(\frac{1}{r^{2}}+\frac{\nu^{\prime}}{r}\right)=-8 \pi p+E^{2}, \\
G_{2}^{2}=e^{-\lambda}\left[\frac{1}{4} \nu^{\prime} \lambda^{\prime}-\frac{1}{4} \nu^{\prime 2}-\frac{1}{2} \nu^{\prime \prime}-\frac{1}{2}\left(\frac{\nu^{\prime}-\lambda^{\prime}}{r}\right)\right]=-8 \pi p-E^{2} .
\end{gathered}
$$

上列各式中 $E^{2}$ 由式(2)求解得 $E^{2}=\frac{Q^{2}(r)}{r^{4}}, Q(r)$ 为 $r$ 内部球的电荷, 在均匀电荷密度下 $E^{2}=$ $h^{2} r^{2}$.

在 $r$ 内部的球质量 $m(r)$ 包含两部分, 即

$$
m(r)=m_{0}(r)+m_{i}(Q(r)),
$$

式中纯引力质量 $m_{0}(r)=4 \pi \int \rho_{0} r^{2} d r, m_{c}(Q(r))$ 即电磁质量.

求解式 (3) 可得

$$
e^{\lambda}=\left(1-\frac{2 m(r)}{r}-\frac{2 \eta(r)}{r}\right)^{-1},
$$

本文 1981 年7月 29 日收到。 
$\eta(r)=\frac{B}{2} \int \frac{Q^{2}(r)}{r^{2}} d r, B$ 是和电荷分布有关的常数.

将式(4)求导, 并由式(3)、(4)和(5)可得

$$
p^{\prime}(r)=\frac{Q(r) \rho_{e}}{r^{2}}-\frac{v^{\prime}}{2}\left(\rho_{0}(r)+p(r)\right),
$$

从上式和场方程求解可得内部解的一般形式

$$
\begin{aligned}
e^{v}=\exp \left\{-\int_{r}^{\infty} \frac{2}{r}\right. & {\left[m(r)+4 \pi r^{3} p(r)+\eta(r)-\left(1-\frac{B}{2}\right) \frac{Q^{2}(r)}{r}\right] } \\
& \left.\left(1-\frac{2 m(r)}{r}-\frac{2 \eta(r)}{r}\right)^{-1} d r\right\} .
\end{aligned}
$$

另外, 我们可得推广的 $\mathrm{T}-\mathrm{O}-\mathrm{V}$ 方程如下:

$$
\begin{gathered}
\frac{d p}{d r}=\frac{Q(r) \rho_{e}}{r^{2}}-\frac{m(r) \rho}{r^{2}}\left[1+\frac{4 \pi r^{3} p(r)}{m(r)}+\frac{\eta(r)}{m(r)}-\left(1-\frac{B}{2}\right) \frac{Q^{2}(r)}{r m(r)}\right] \\
\left(1+\frac{p(r)}{\rho}\right)\left(1-\frac{2 m(r)}{r}-\frac{2 \eta(r)}{r}\right)^{-1},
\end{gathered}
$$

此即球对称理想流体内部静压平衡方程. 其形式较 Bekenstein 的形式多一项与 $B$ 有关的项.

\section{二、均匀密度的内部解}

在 $\rho_{e}=$ const 的均匀电荷分布下, 电磁质量为 ${ }^{[1]} \frac{3}{5} \frac{Q^{2}(r)}{r}$, 于是 $(6)$ 式中的总质量 $m(r)=$ $4 \pi \int \rho r^{2} d r$ 为

$$
m(r)=m_{0}(r)+\frac{3}{5} \frac{Q^{2}(r)}{r}
$$

从上式可得总质量密度 $\rho$ 为

$$
\rho=\rho_{0}+\frac{3}{4 \pi} h^{2} r^{2}
$$

从上式可见总质量密度 $\rho$ 包含纯引力质量密度 $\rho_{0}$ 和电磁质量密度 $\frac{3}{4 \pi} h^{2} r^{2}$ 两部分. 我们采用 的均匀密度是指 $\rho=$ const, 此时 $\rho_{0}=\rho-\frac{3}{4 \pi} h^{2} r^{2}$, 即纯引力质量密度 $\rho_{0}$ 不是均匀分布的, 但 总质量密度是均匀分布. 在场方程式(3)中出现的密度其物理含义应指纯引力质量密度 $\rho_{0}$, 若 将其换成总质量密度 $\rho$ 可把式(12)代人式(3)得

$$
e^{-\lambda}\left(\frac{1}{r^{2}}-\frac{\lambda^{\prime}}{r}\right)-\frac{1}{r^{2}}=-8 \pi \rho+5 h^{2} r^{2},
$$

从上式可见式(7)中 $B=-5$. 求解上述方程可得

$$
e^{\lambda}=\left(1-\frac{r^{2}}{R^{2}}+h^{2} r^{4}\right)^{-1}
$$

上式显然与式(1)不同, 上式中 $R^{2}=\frac{3}{8 \pi \rho}$, 与总质量密度有关. 但式 (1) 是 $\rho_{0}=$ const 的内部 解, 此时总质量密度 $\rho$ 不是均匀分布的. 而式 (13) 是 $\rho=$ const 的内部解. 可证明式 (1) 和

第 3 期

科学通报 
式(13)均符合边界条件, 即都和 Reissner-Nordström 外部解在边界面上相吻合. 但两者是在不 同均匀密度定义下的内部解。

\section{三、荷电球内部解的共形唯一性}

下面我们将证明, 内部解式 (13) 是共形平直内部解, 而内部解式 (1)不是共形平直的, 即式 （13）满足共形唯一性要求.

由 Ricci 张量 $R_{1}^{1}$ 和 $R_{2}^{2}$ 之差, 我们有

$$
e^{-\lambda}\left[\frac{\nu^{\prime \prime}}{2}-\frac{\lambda^{\prime} \nu^{\prime}}{4}+\frac{\nu^{\prime 2}}{4}-\frac{1}{r^{2}}-\frac{1}{2 r}\left(\lambda^{\prime}+\nu^{\prime}\right)+\frac{e^{\lambda}}{r^{2}}\right]=2 E^{2} .
$$

另外,由共形平直时 Weyl 张量 ${ }^{[7]}$ 为零有

$$
\frac{e^{\lambda}}{r^{2}}-\frac{1}{r^{2}}-\frac{\nu^{\prime 2}}{4}+\frac{\nu^{\prime} \lambda^{\prime}}{4}-\frac{\nu^{\prime \prime}}{2}-\frac{1}{2 r}\left(\lambda^{\prime}-\nu^{\prime}\right)=0 .
$$

将式(14)两边乘以 $e^{\lambda}$ 后和(15)相加可得

$$
\frac{1}{r^{2}}-\frac{\lambda^{\prime}}{2 r} e^{-\lambda}-\frac{1}{r^{2}} e^{-\lambda}=h^{2} r^{2},
$$

将式(1)代入式(16)时上述恒等式不能成立. 将 $e^{\lambda}$ 取式(13)代入式 (16) 后恒等式成立, 或反 之, 求解式(16)可得式(13). 这说明式(1)不是共形平直的, 而式(13)是共形平直内部解, 它满 足共形唯一性.

\section{四、均匀密度共形平直内部解}

我们现在精确求解共形平直内部解的 $e^{\nu}=g_{t t}$ 的形式. 从式(3)和式(4)经运算后得

$$
e^{-\lambda} v^{\prime}-\left(e^{-\lambda}\right)^{\prime}=8 \pi r\left(\rho_{0}+p\right),
$$

另一方面,我们求解(8)式可得

$$
p(r)+\rho_{0}=e^{-\frac{1}{2} \nu} \int \frac{Q(r) \rho_{e}}{r} e^{v / 2} d r,
$$

冷 $y=e^{v / 2}$, 将(18)式代入(17)式,可得下列微分方程

$$
\begin{gathered}
\left(1-\frac{2 m(r)}{r}-\frac{2 \eta(r)}{r}\right) y^{\prime}-\frac{1}{2}\left(8 \pi r \rho_{0}+\frac{2 m(r)}{r^{2}}-\frac{B Q^{2}(r)}{r^{3}}+\frac{2 \eta(r)}{r^{3}}\right) y \\
=4 \pi r \int \frac{Q(r) \rho_{e}}{r^{2}} y(r) d r .
\end{gathered}
$$

在均匀密度时, 从式 (13) 可将上式化为

$$
\left(\frac{1}{r}-\frac{r}{R^{2}}-h^{2} r^{3}\right) y^{\prime \prime}+\left(-\frac{1}{r}+h^{2} r^{2}\right) y^{\prime}-h^{2} r y=0,
$$

此方程是 Fuchs 型微分方程, 在数学上共有五个奇点, 三个是实数, 二个是虚数, $r$ 必须是实数 才有物理意义. 三个实数奇点中除 $r=0$ 处外尚存在两个座标奇点,即

$$
r=\sqrt{\frac{1 \pm \sqrt{1-4 h^{2} R^{4}}}{2 h^{2} R^{2}}},
$$

可以证明,这两个内部解的座标奇点在边界处和 $R-N$ 外部解的座标奇点相吻合.

令 $r^{2}=u$, 式 $\left(19^{\prime}\right)$ 变为 


$$
\left(1-\frac{u}{R^{2}}+h^{2} u^{2}\right) \frac{d^{2} y}{d u^{2}}+\frac{1}{2}\left(-\frac{1}{R^{2}}+2 h^{2} u\right) \frac{d y}{d u}-\frac{1}{4} h^{2} y=0,
$$

用类似于文献 $[6]$ 的变量代换法, 将 $u$ 变换成 $v$

于是式(21) 化为

$$
v=\frac{1}{2} \sin ^{-1}\left(\frac{1-2 h^{2} R^{2} u}{\sqrt{1-4 h^{2} R^{4}}}\right),
$$

$$
\frac{d^{2} y}{d v^{2}}+y=0
$$

式(23)的一般解为

$$
y=a_{1} e^{i v}+a_{2} e^{-i v}=A_{1}^{1 / 2} \sin \left(v+A_{2}\right),
$$

此处 $A_{1}$ 和 $A_{2}$ 是积分常数, 于是

$$
e^{v}=y^{2}=A_{1} \sin ^{2}\left(v+A_{2}\right) .
$$

另一方面, 从式(4)、(13)和 (24) 我们可求得压强 $p$ 的表式为

$$
p=\frac{h^{2}}{4 \pi} r^{2}-\frac{\rho}{3}-\frac{h}{4 \pi} \frac{\left(1-r^{2} / R^{2}+h^{2} r^{4}\right)^{1 / 2}}{\tan \left[\frac{1}{2} \sin ^{-1}\left(\frac{1-2 h^{2} R^{2} r^{2}}{\sqrt{1-4 h^{2} R^{4}}}\right)+A_{2}\right]} .
$$

两个积分常数 $A_{1}$ 和 $A_{2}$ 可从边界条件来定, 首先, 由 $r=r_{0}$ 处压强为零可得

$$
A_{2}=-\frac{1-2 h^{2} R^{2} r_{0}^{2}}{\sqrt{1-4 h^{2} R^{4}}}+\sin \left\{2 \tan ^{-1}\left[\frac{h\left(1-r_{0}^{2} / R^{2}+h^{2} r^{4}\right)^{1 / 2}}{h^{2} r_{0}^{2}-\frac{1}{2} R^{2}}\right]\right\} \text {, }
$$

然后由 $e^{v}$ 在边界处连续要求可得

$$
A_{1}=\left(1-\frac{r_{0}^{2}}{R^{2}}+h^{2} r_{0}^{4}\right)\left\{\sin \left[\frac{1}{2} \sin ^{-1}\left(\frac{1-2 h^{2} R^{2} r_{0}^{2}}{\sqrt{1-4 h^{2} R^{4}}}\right)+A_{2}\right]\right\}^{-2} \text {. }
$$

将式 (22) 代入式 (24) 可得

$$
e^{v}=A_{1} \sin ^{2}\left[\frac{1}{2} \sin ^{-1}\left(\frac{1-2 h^{2} R^{2} r^{2}}{\sqrt{1-4 h^{2} R^{4}}}\right)+A_{2}\right],
$$

我们的形式不同于 Mehra 和 Bohra ${ }^{[6]}$ 的形式. 由式(28)可见 $r$ 必须满足下列条件,此解才有 意义。

$$
-1 \leqslant \frac{1-2 h^{2} R^{2} r^{2}}{\sqrt{1-4 h^{2} R^{4}}} \leqslant 1
$$

或将 $h^{2}=\frac{Q^{2}}{r_{0}^{6}}$ 和 $R^{2}=\frac{3}{8 \pi \rho}=\frac{r_{0}^{3}}{2 M}(Q$ 和 $M$ 分别为球体总电荷和总质量, $)$ 代人式 $(2 \circ)$ 可得类似 的条件

$$
-1 \leqslant \frac{1-\frac{Q^{2}}{M r_{0}^{3}} r^{2}}{\sqrt{1-\frac{Q^{2}}{M^{2}}}} \leqslant 1,
$$

从式(30)我们可见, 内部解只有满足下列条件才有意义.

$$
M^{2}>Q^{2} \text {. }
$$

反之, 若 $M^{2}<Q^{2}$ 则内部解无意义, 即库仑厉力大于引力时球体将是膨胀的动态解, 不可能存 
在静态解. 另外, 从式(29)可见 $r$ 必须在下列范围内才有意义

$$
\sqrt{\frac{1+\sqrt{1-4 h^{2} R^{4}}}{2 h^{2} R^{2}}} \geqslant r \geqslant \sqrt{\frac{1-\sqrt{1-4 h^{2} R^{4}}}{2 h^{2} R^{2}}},
$$

式 (32) 的两边和式 (20) 相同, 它们和 $R-N$ 度规的两个坐标奇点相对应. 由式 (32) 可见,式 （28）的内部解在荷电黑洞情况下是无意义的, 这是由于黑洞内部不可能存在确定的静态。换 言之,若不是荷电黑洞,则在满足式(31)和 $r$ 在式(32)范围内式(28)内部解是存在的.

致谢：作者对美国与里兰大学的胡比乐教授和犹他大学的 Mashhoon 教授以及日本京都大学的佐藤文 隆教授以及郭汉英、阙仲元和冯正良等先生的邦助和讨论深表感谢.

\section{参考文献}

[1] Bonner, W. B., Z. Physik, 59(1960), 160.

[2] Efinger, H. J., Z. Physik, 31(1965), 188.

[3] Wilson, S. J., Can. J. Phys., 47(1967), 2401.

[4] Bailyn, M. \& Eimarl, D., Phys. Rev., D5(1972), 1897; Bailyn, M., Phys. Rev., D8(1973), 1036.

[5] Bekenstein, J. D., Phys. Rcv., D4(1971), 2185.

[6] Mehra, A. L. \& Bohra, M. L., GRG, 11(1979), 333.

[ 7] Gürses, M. \& Gürscy,'Y., Nuovo Cimento, 25B (1975), 786. 